\title{
Influencia de la página de Facebook de la Universidad Politécnica Salesiana, sede Guayaquil, como medio de información para los estudiantes
}

\section{Influence of the Facebook page of the Universidad Politécnica Salesiana, Guayaquil headquarters, as a means of information for students}

Betty Isabel Rodas

Universidad Politécnica Salesiana, Ecuador

Ronald Álvarez

Universidad Politécnica Salesiana, Ecuador

Autor para correspondencia: brodas@ups.edu.ec

Fecha de recepción: 30 de Agosto de 2016 - Fecha de aceptación: 01 de Noviembre de 2016

Resumen: El avance tecnológico mediatiza y transforma la comunicación, digitalizando el modo tradicional y material de informarse. Se yuxtaponen los encuentros físicos por las nuevas plataformas, dentro de la web 2.0, donde se gesta la sociedad en los ámbitos de identificación e información, no solo como escenario de interacción sino también un espacio para comunicarse. Desde este contexto, la Universidad Politécnica Salesiana sede Guayaquil, en el año 2010, crea una plataforma en la conocida red social Facebook, con el propósito de presentar un espacio de identidad y abastecimiento de noticias dentro de la comunidad universitaria para que los estudiantes puedan acceder a la información de manera oficial. En consecuencia, la investigación analiza el impacto y contribución en el desarrollo académico, con el fin de constatar la intencionalidad de su creación, los contenidos que se difunden, los niveles de pertinencia en el consumo y la factibilidad de su continuidad, como medio de información, interacción e identidad en los estudiantes.

Palabras claves: tecnología; información; comunicación; facebook; interactividad

Abstract: Given the need for professorial training process in Education Science and its social significance, the objective of this work is to reflect on the training in university teaching from its application in practice. From the theoretical foundations of the educability and educatividad, an interactive program that would combine presentiality using virtual resources, was conceived through a course that would privilege the professional exchange with the possibilities of using the Virtual Classroom Cooperative Learning (AVAC) of the University. It was intended to enable socialization and assimilation cooperatively content, paying special attention to the theory linking practice. In the evaluative plane it was helped to reaffirm the sense of belonging to the teaching profession, regardless of the subjects head into the teaching-learning process. 94\% of participants said the positive impact of the ongoing transformation of teaching. In this area the significance of the teaching work to finalize highlighted the links between science and subject to contribute to the integral formation of students, in the framework of the substantive processes of higher education. Key words: technology; information; communication; facebook; interactivity 


\section{Introducción}

La estructura comunicacional y el intercambio de información, han evidenciado a lo largo de su evolución histórica, una serie de contradicciones, según García ${ }^{1}(2002)$ considera que el desarrollo de la humanidad, la descapitalización del conocimiento o antagónico a ello, el esclarecimiento de la fuente de la información y del sujeto de su dependencia (lo cual se podría comprender dentro de la microfísica del poder propuesta por Foucault), tiñe lo social en su conjunto. Por tanto, el conocimiento tiene la imperiosidad de difundirse, y en conjunto la información converge un sinnúmero de factores que inciden directamente en una sociedad mediatizada. Se advierte así que, desde la escritura en papel a la imprenta de Gutenberg en 1455, y posteriormente la revolución de la imprenta con el advenimiento de los primeros libros llamados los incunables, el cambio y la praxis de la información, ha evidenciado una innovación en cuanto a su transmisión, similar a la Revolución Industrial de finales del siglo XVIII e inicios del XIX (Drucker, 2001).

La revolución industrial, trajo aparejados inventos y transformaciones, la máquina de vapor renovó el proceso comunicacional acortando tiempo y espacio a la difusión y al comercio; que más tarde permitieron la creación del telégrafo eléctrico y la fotografía que llegaron hacia el año de 1830 (Drucker, 2001) y que también marcaron un hito, no solo en el evidente desarrollo de la tecnología, sino en la difusión de información y en el desplazamiento del conocimiento compartido. A la par de los cambios tecnológicos se suscitan levantamientos, tanto individuales como sociales, jerárquicas y políticas, informativas y comunicativas, que no dejaron esperar en el ser del sujeto; la accesibilidad de la información, las incoherencias de la misma, la facilidad del diálogo y la ruptura de distancias, son algunos de los hechos que revolucionaron el quehacer social y trasformaron las miradas de los analistas en casos situacionales de este estilo.

Para March (2012) acuñando el concepto de Augé $^{2}$ involucra a la sociedad del siglo XX mencionándola como la modernidad porque se refirió a la aceleración de procesos acontecidos en el siglo comprendido del XVII al XIX, época que propicio el nacimiento de conceptos para el estudio relacionados al análisis de la información. Mediante la individualización colectiva, las sociedades buscaron asociarse con los entornos a pesar que la historia no mostraba dichos avances, pero se encontraba en constante desarrollo. Con el devenir del tiempo, las personas se encuentran más informadas de los acontecimientos que suceden alrededor del mundo buscando sus intereses de información, dejando de lado formas tradicionales de adquisición de datos, información y conocimiento, evidenciando nuevos espacios de desarrollo comunicativo y social.

La forma de transmisión en el plano tradicional, establece un emisor único y un receptor que espera pasivamente, datos un modelo mecanicista el cual discrepa y queda sustituido por el paradigma informativo, en el cual el mismo receptor puede mudarse de rol transformándose en un emisor, de diversas recepciones. La era actual se encuentra regida por información inmediata y factible, gestándose estos como los principales factores que dominan la comunicación humana, por ende, la sociedad se va constituyendo en un proceso de inevitable evolución tecnológicacomunicacional y, la facilidad y accesibilidad de la misma, le permiten al sujeto generar intereses

\footnotetext{
${ }^{1}$ García María Inés, investigadora de origen argentino reside actualmente en México, se ha dedicado a estudiar y analizar los pensamientos Michel Foacault.

${ }^{2}$ Marc Augé: Antropólogo francés nacido en Poitiers, especializado en la disciplina etnológica.
} 
particulares en el basto cúmulo de información.

La continuidad evolutiva de la tecnología, lleva consigo consecuencias sociales, entre ellas las comunicativas, que evolucionaron en ese despegue de la transmisión informativa que trajo el internet; así el desarrollo de los espacios de conocimiento en internet desde la primera generación 1.0, considerando prototipos de interacción y sustitución de la información materializada, que aunque estáticos y cerrados a la manipulación del público, generaban una puesta en escena de la nueva información digital, que más tarde se desarrollará, en una plena y completa interacción, dentro de la web 2.0.

El inconmensurable número de personas que se suman a los espacios virtuales y que despliegan nuevas interacciones sociales, por lo general ya no se vuelcan a los medios de comunicación clásicos, sino a estos puntuales espacios que incrementan millonarias cantidades, extendiendo de ese modo su publicidad y logrando la personalización de los usuarios que rompen con algunas de las barreras de las viejas organizaciones mediáticas (Campos, 2008), respaldados por la enorme cantidad de información y conocimiento acumulado en "el universo llamado internet”, que contempla el declive de una agónica desaparición del tradicionalismo comunicacional e informativo.

Pese a que la mediatización comunicativa genera incertidumbres por el posicionamiento de la información, este nuevo desarrollo informativo no escapa de las mismas, que lejos de ser diferentes, presenta similares realidades para cuestionar, como la sensatez de la información, los mecanismos de regulación o la verosimilitud de la realidad, que en la actualidad genera múltiples estudios, entrando quizás a una nueva paradoja del capital, que está por encima de todo, denominada el poder global de la red y la tecnología que poco a poco se va creando, que ofrece prosperidad y desigualdad, creatividad y desintegración (Castells, 2001). Con todo lo antes expuesto, se puede apreciar, que el internet es el presente y un expectante futuro, no solo para la información sino para el general desarrollo social, que en palabras de Castells (2001) señala:

Internet es el tejido de nuestras vidas. No es futuro. Es presente. Es un medio para todo. Internet es la sociedad, expresa los procesos sociales, los intereses sociales, los valores sociales, las instituciones sociales. Su especificidad es que constituye la base material y tecnológica de la sociedad red (p. 1).

Al respecto Drucker (2001) se pronuncia como el impacto que puede ser aún mayor en las sociedades y en la política y, sobretodo, en el modo en que se configura el mundo, desde este contexto la investigación centra su mirada en el punto de la influencia que puede ejercer la web $2.0^{3}$, específicamente la red social Facebook en un espacio particular, la Universidad Politécnica Salesiana- Guayaquil, como medio de información en un grupo universitario.

Con la premisa expuesta con anterioridad, se requiere comprender la realidad del país dentro de la evidente postura comunicativa que se está desarrollando y que se va alojando dentro del sector "Facebook" como medio primigenio de interacción. Para ello se considera importante presentar este trabajo, con una mirada deductiva, sobre la reacción e influencia de esta red social,

\footnotetext{
${ }^{3}$ Web 2.0 mejor conocida como Web Social, engloba un gran número de cibernautas como comunidad para interacción dentro del internet y sus múltiples plataformas.
} 
en la Universidad Politécnica Salesiana sede Guayaquil, como un espacio de nueva información que procese de manera inmediata los hechos y desarrollos que se vayan gestando en los ambientes de la institución, teniendo en cuenta el evidente cambio virtual que existe para las nuevas plataformas de interacción social y desarrollando con ello una visión posicional del pensamiento, elección y desarrollo colectivo virtual-comunicacional.

Así, esta investigación, presenta en un primera instancia, un análisis histórico, de las diversas interacciones comunicativas, es decir; observa la tecnología y sus cambios influyen de forma significativa, no solo en la comprensión de una sociedad, sino en el entorno en el cual se desarrolla, puntualizando la idea dentro de los medios comunicativos, con lo que implica la información, el entretenimiento y más; teniendo en cuenta un correcto planteamiento metodológico, sustentado en documentaciones que analicen los patrones de evolución, y con ello los niveles de consumo. Es fundamental ampliar esta idea inicial, pues la necesidad comunicativa del hombre es inherente a su ser, y con el paso del tiempo siempre se gestan nuevos procesos con los cuales se mejore esta facultad comunicativa. El avance en la comunicación se va desarrollando a tal punto, que en la actualidad, nos vemos inmersos en un inmediatismo informativo, que produce no solo una comunicación instantánea, sino también un cúmulo de información infalible.

Los inevitables cambios tecnológicos, que van de la mano del desarrollo comunicativo, catapultan la investigación, hacia un hecho concreto, la influencia que puede suscitar la red social, particularmente la red social Facebook, en un grupo específico universitario, que representa el universo del trabajo. En este segundo punto del estudio, se analiza cual es el tipo de interés e influencia, que promueva el espacio Facebook de la Universidad Politécnica Salesiana sede Guayaquil, en estudiantes, comprendiendo que, en la actualidad, la sociedad se gesta mediante las nuevas redes de interacción comunicativa.

Por tanto este artículo, analiza la valoración e influencia de la información en la comunidad universitaria desde la red social Facebook. Para medir la propuesta de intencionalidad de su creación, los contenidos que se difunden, los niveles de pertinencia en el consumo y las póstumas propuestas a desarrollarse en torno a la factibilidad de su continuidad, como medio de información, interacción e identidad en los estudiantes.

Se trata de mostrar si la red social Facebook, como espacio de información, de conocimiento e identidad, está cumpliendo con ese fin específico; de esta manera evidenciar el grado de su funcionalidad y el aceptable apogeo de su continuidad. Este estudio, pretende crear una reflexión que se inmiscuya de forma objetiva, hacia una temática extensa y compleja, de nuevas investigaciones en el marco de un correcto desarrollo y análisis comunicacional.

\section{Métodos}

El tratamiento de la investigación se desarrolla mediante un análisis de carácter deductivo, conceptual, histórico, que utiliza como instrumento a la encuesta, convirtiéndolo así, en un estudio pluriparadigmático. Referente al proceso pluriparadigmático, Pérez \& Porras (2005), mencionan que: "se cree firmemente en la posibilidad de existencia de una multiplicidad de paradigmas, que a su vez potencian el surgimiento de una trama de interacciones entre los 
estatutos epistemológicos y las metodologías emergentes" (p. 105), aludiendo que, no solo es importante investigar con esta mirada, sino más bien es necesario y es fundamental utlizar este modelo metodológico.

Todo este proceso metodológico, gesta un análisis particular, en el marco de la inmensa multiplicidad de estudios que se pueden suscitar en el extenso universo de investigaciones de las nuevas interacciones sociales dentro de la web 2.0, analizando un fenómeno global vigente en el desarrollo comunicativo, que especifica detalles muy importantes del sector a investigar, más aún si el grupo es una considerable comunidad universitaria. Comprendiendo con ello la importancia de generar un trabajo descriptivo y de exploración de documentos (técnicas elementales), libros y revistas indexadas; que permitan crear procedimientos adecuados para la conmensuración de ideas dispersas y la futura concatenación de elementos trascendentales, entorno a la realidad investigativa, se muestra en este espacio todas las consideraciones metodológicas utilizadas.

El inicio de esta investigación trajo consigo varios cuestionamientos, respecto al hecho de comprender y evaluar la influencia que tiene la plataforma Facebook de la UPS-Guayaquil, en un sector específico de la comunidad universitaria, para ello identificar la población y muestra para realizar el trabajo de campo constituyó un factor preponderante. Así, se puede vislumbrar en la investigación, que el universo del trabajo se centra en cursos específicos de la universidad, que presenten distintos tipos de resultados y a su vez, ejemplifiquen toda la magnitud que comprende la institución. Esto quiere decir que los cursos son la voz de la universidad, en este caso, pudiendo ser también la voz de un colectivo que se encuentra inmerso, comunicado e influido en los nuevos espacios de interacción social.

Por consiguiente, se procedió a solicitar el informe oficial de estudiantes legalmente matriculados por la Secretaria de Campus de la UPS-G (periodo 46), resaltando que la presente investigación inició desde el mes de mayo del 2015 con el periodo 46 y trabajar inmediatamente en él. El detalle fue facilitado por la Lcda. Karla Sofía Romero Quiroz, Secretaria General de la sede. En el documento que se anexa a la investigación, se evidencia que existen 7.722 estudiantes, por tal motivo, se ha centrado el punto de atención a éste número, para obtener la muestra y realizar las entrevistas en base al porcentaje de la población que se provee la UPS-G, bajo juicio de experto.

En consecuencia y con el propósito de buscar sujetos idóneos para la investigación, se optó por el muestreo probabilístico, aplicando la fórmula proporcionada por el experto, economista Víctor Javier Arcos, docente de la UPS-G, la muestra la calculó a través de (Corporacion Asesoría Económica \& Marketing, 2009), seleccionado aleatoriamente los grupos de las carreras existentes de la comunidad universitaria: Ingeniería en Sistemas, Ingeniería Industrial, Ingeniería Electrónica, Ingeniería Eléctrica, Administración de Empresas, Contabilidad y Auditoría y Comunicación Social, para la ejecución de las encuestas y de esta forma logar la representatividad necesaria, el detalle del cálculo de la muestra se presenta a continuación :

Registro oficial estudiantes matriculados UPS-G.

Margen de Error: 5\%

Nivel de Confianza: $95 \%$ 
Tamaño de Población: 7.722

Tamaño de Muestra: $\underline{366}$

Ecuaciones estadísticas para proporciones poblacionales.

$\mathrm{n}=$ Tamaño de la muestra

$\mathrm{z}=$ Nivel de confianza deseado

$\mathrm{p}=$ Proporción de la población con la característica deseada (éxito)

$\mathrm{q}=$ Proporción de la población con la característica deseada (fracaso)

$\mathrm{e}=$ Nivel de error dispuesto a cometer

$\mathrm{N}=$ Tamaño de la población

$$
\mathrm{n}=\frac{\mathrm{z}^{2}(\mathrm{p} * \mathrm{q})}{\mathrm{e}^{2}+\frac{\mathrm{z}^{2}(\mathrm{p} * \mathrm{q})}{\mathrm{N}}} \quad \mathrm{n}=\frac{1.96^{2}(0.5(0.5))}{0.05^{2}+\frac{1.96^{2}(0.5(0.5))}{7722}} \quad \mathrm{n}=\frac{0.9604}{0.0026243} \quad \mathrm{~N}=366
$$

Se desprende que el trabajo de campo se desarrolló con una muestra de 366 estudiantes del total de la población, paralelamente y acorde con el carácter pluriparadigmático de la investigación; es preciso acotar desde este tamiz y tomando como referente los antecedentes planteados en relación a la población y muestra los métodos, utilizados (conforme al estudio pluridigmàtico) de esta forma el método histórico - lógico permite analizar las etapas que se van suscitando, por un lado, en el desarrollo tecnológico para cambiar los modelos de comunicación y por el otro las diferentes influencias que se suscitan del mismo.

Se considera trascendental este método debido a su incidencia en la comprensión de los cambios, los cuales traen aparejados nuevos conocimientos, después de una revolución cuando se comprende un cambio, y después de un cambio que da frutos a nuevos acontecimientos, resultado de un adagio popular de las guerras clásicas; puesta en escena sobre la realidad que se conjetura estudiar de manera deductiva, traslada la metáfora, con el tratamiento de la investigación, comprendiendo que la revolución tecnológica reconoce un evidente cambio social, alterando las presunciones de estabilidad y enfrentamientos de la coyuntura tradicional con el acelerado cambio de la misma. Es innegable que la revolución tecnológica altere y trastoque específicos puntos inherentes al sujeto como se da en el caso de la comunicación, desplegando cada vez más y mejores formas de hacerlo.

Asumiendo que los hechos se construyen en la historia para revelar orígenes, continuidades, rupturas, caminos ya transitados, líneas abandonadas o problemas no bien explorados pues esto es lo que puede aportar una mirada histórica, y es ahí donde historia y crítica de la investigación tienen un espacio de encuentro (Martínez, 2009). El proceso con el cual se va desarrollando esta información analiza el hecho de las actividades de cambio que van produciendo con el ingreso de los nuevos procesos de comunicación.

Las redes sociales ${ }^{4}$ y la web 2.0, particularizando a Facebook, tiene un alto índice de

\footnotetext{
${ }^{4}$ Redes sociales: Espacios sociales dentro de las múltiples plataformas (web 2.0) en la que interactúan varios usuarios.
} 
aceptación y consumo en Ecuador. Un grupo de investigadores del Oxford Internet Institute (OII, 2015) en base a investigaciones tomó de Alexa $^{5}$ datos provistos por el sitio estimando cuáles son los sitios más populares de cada país. Los resultados son indiscutibles ya que Google y Facebook se reparten el mapa casi en partes iguales, aunque el buscador muestra una leve ventaja sobre la red social, en este análisis se puede determinar cómo los datos se vislumbran con un mapamundi, donde determina el tamaño de cada país sobre la base de su población de usuarios de Internet (LA NACION, 2013), mostrando de ese modo como los ecuatorianos acogen con mucha fascinación las redes sociales y en especial Facebook, ubicándolo en el segundo lugar del top site $^{6}$ con acceso restringido a cifras. Otro de los métodos empleados es el de Inducción y deducción, procesos que suponen sustancialmente dos instancias en cuanto al tratamiento del contenido de forma particular y general a la vez, y es así como el progreso de la investigación tiene en su causal estas dos metodologías primando la idea de comprender que el estudio en torno a las redes sociales, tiene un universo infinito de investigaciones y aristas por estudiar.

Con el proceso Inductivo, se debe comparar lo que plantea Chalmers (1987), al mencionar que el proceso de induccion "nos lleva de una lista finita de enunciados singulares a la justificación de un enunciado universal, que nos lleva de la parte al todo, se denomina razonamiento inductivo y el proceso se denomina inducción" (p.6), así es importante resolver la importancia de los resultados generales que pueden orienar esta particularidad.

Por su parte se comprende que el método dedutivo, se contrapone al planteamiento mostrado con anterioridad, generando con ello, de un grupo máximo (o general) una visión hacia lo particular.

Esto demuestra que una vez que la investigacion tiene a su disposición leyes y teorías universales puede extraer de ellas diversas consecuencias que le sirven como explicaciones y predicciones (Chalmers, 1987) y con ellas analizar las posibilidades de estudio que se generan en sectores, individuos o lugares particulares, anailzando la influencia de algo universal en algo simple. Hace mención el alto contenido de informacion que se necesitaba disernir para trabajar de forma puntual en esta investigación.

Se recurre también al método cualitativo, el cual se pone de manifiesto mediante el análisis documentaciones bibliográficas, para no solo unir ideas, sino plasmar un proceso constructivo que permita hacer de esta investigación una futura herramienta de trabajo. En cuanto a la práctica del método cuantitativo, este tendrá lugar para la comprensión del análisis objetivo de los datos investigados en el tratamiento de la información de las encuestas.

\section{Resultados}

Contextualizando la realidad ecuatoriana y latinoamericana, se analizan algunas estadísticas sobre niveles de consumo de internet y de redes sociales presentan una realidad global del incremento del uso de estos medios y del avance participativo por necesidades sociales, de implementación de elementos, aplicación de programas 2.0 y de posibles visiones futuras que ofrecen un nivel de análisis estadístico. En al caso ecuatoriano, pese a que el acceso a

\footnotetext{
5 Alexa: www.alexa.com/topsites/category/World/.../Base_de_datos.

${ }^{6}$ Top site Alexa: http://www.alexa.com/topsites/countries/EC
} 
internet no es una prioridad, incluso presenta limitantes, como en otros países más desarrollados, el uso de las redes sociales incrementa de forma admirable.

Para analizar de forma empírica lo antes mencionado, las estadísticas del INEC, en un análisis del 2013, desglosan la realidad del país, considerando la adquisición de aparatos tecnológicos, el consumo de internet entre otros: En cuanto al alcance de consumo de los medios de tecnología a nivel estudiantil deberá tomar en cuenta datos y comparar el consumo interno a partir de datos generales, la CEPAL (2013) señala que Ecuador tiene 5\% de consumo de internet a nivel mundial porque la mayoría de los hogares no logra ingresos suficientes para acceder a un nivel mínimo de consumo de masas. No sólo del bienestar material, prestigio social y de integración crecientemente valorada por la sociedad. En general sólo dos y tres de cada diez hogares urbanos registran un ingreso per cápita para integrarse a la sociedad de consumo que corresponde al grado de progreso latinoamericano, el consumo será analizado a partir de esta penetrabilidad digital.

Las redes sociales son consideradas un medio de información activa, tiene alcance en los medios de información trascendental en la web según las tendencias digitales expuestas en el INEC (2013) del Guayas tiene un alcance de 41,7\% de la población, la referencia del estudio se realizará considerando la brecha digital.

Observando el aporte que realiza los ecuatorianos en el crecimiento de la web 2.0, se conoce que suben muy pocos contenidos a la Internet, en comparación con los cibernautas de la región latinoamericana. Esto está ligado a la capacidad tecnológica del país, al ancho de banda, el costo de la conexión y el aprovechamiento de los 'smartphones ${ }^{7}$, , según la encuesta Wave $7^{8}$, que se realizó a inicios de este año en 54 países del mundo y que obtuvo datos de más de 1.000 millones de usuarios de Internet (El Comercio, 2014), sin embargo las encuestas también muestran de manera global que el incremento en el uso del internet, específicamente de las redes sociales, ha acrecentado de forma significativa.

Para la Wave 7 (2013), hay motivaciones específicas de los usuarios de internet ${ }^{9}$, que han estado estudiadas durante siete años, estas son: relación, diversión, progreso, reconocimiento y aprendizaje. El resultado para comprenderlo se ve en el evidente consumo e interacción en las redes sociales, tengan el propósito que sea en los individuos particulares, podremos deducir con las estadísticas que, no hay joven sin la intervención de estas redes en su vida, o al menos una inmensa mayoría cubre la realidad de un pequeño grupo anacrónico que no las utiliza; para la plataforma de análisis web estatista señala que: "el número de usuarios de redes sociales en el mundo en 2010 fue de 970 millones y la proyección al 2014 sean 1.820 millones" (El Comercio, 2014).

El INEC (2013) presenta la realidad de los ecuatorianos, mencionando más hombres que mujeres, y que ellos utilizan las redes principalmente para comunicarse, informarse, educarse y

\footnotetext{
7 Smartphones: El término pertenece a la lengua inglesa y hace referencia a aquello que, en nuestro idioma, conocemos como teléfono inteligente. Se trata de un teléfono celular (móvil) que ofrece prestaciones similares a las que brinda una computadora (ordenador) y que se destaca por su conectividad.

${ }^{8}$ Wave 7: Examinador de smartphones, uso e ingreso de usuarios a la internet.

${ }^{9}$ Internet: Red informática mundial, descentralizada, formada por la conexión directa entre computadoras mediante un protocolo especial de comunicación.
} 
trabajar. La mayoría de usuarios son jóvenes entre 16 y 24 años, mostrándose este como el perfil o target de personas que navegan por internet. Finalmente, y concatenando los dos procesos, estas convalidan la investigación en un proyecto inductivo que analizará sí el objetivo de la página de Facebook de la UPS-G, lleva de la mano el análisis y aporta la información a los estudiantes de la institución, para mediar el nivel de aceptación y funcionamiento de la misma, para lo cual se observará minuciosamente la incidencia y las evoluciones de la plataforma desde su año de creación hasta la actualidad.

Con los datos estadísticos y evidencias empíricas recabadas se contrasta la información con él la recopilación de información obtenida mediante la aplicación de los instrumentos de las encuestas, los cuales permiten establecer parámetros sobre los factores que inciden en el uso y aplicabilidad de Facebook como una herramienta comunicacional específicamente en la sede Guayaquil de la Universidad Politécnica Salesiana, de este modo los resultados obtenidos, parten del análisis del aspecto edad de los estudiantes, del tiempo de usabilidad al día para revisar información en la página, el conocimiento sobre la existencia de una plataforma que posee información universitaria, la frecuencia de visitas registradas en este espacio en la red. El contenido de esta investigación apunta a identificar posibles cambios positivos al espacio de interacción, ubicado en la red social Facebook, tomando en consideración que deben ser congruentes con las variadas actividades de la institución en el orden académico, cultural y científico.

En consecuencia, mediante la investigación desplegada se determina que en la Universidad Politécnica Salesiana sede Guayaquil (UPS-G), la creación de la página de Facebook surge con la finalidad de ayudar a los estudiantes y al público en general de forma coyuntural en los contenidos informativos que se socializan de este modo el hecho de estar informado sobre los acontecimientos académicos y administrativos es parte de ser Salesianos.

En otro orden de ideas, es importante destacar que a partir del año 2010 como Ups Guayaquil - Educación, comienza asentar sus primeras publicaciones de interés para estudiantes, docentes y administrativos de la UPSG. Nace desde la Dirección Técnica de Comunicación y Cultura, bajo la antigua dirección de la Lcda. Karina Gisselle Hidalgo Loffredo, representante de Comunicación y Cultura de aquel entonces. Ups Guayaquil, empezó como una cuenta de amigos, con un límite de 5000 usuarios y entre los primeros meses del 2015, Facebook solicitó el cambio a página ya que se trataba de una institución académica y pasó directamente a UPS Guayaquil con la categoría de Educación ${ }^{10}$.

A continuación, se analiza la tabulación realizada, los porcentajes que dieron como resultado de la investigación sobre el tipo de servicio que está presentando el espacio de la UPS$\mathrm{G}$ en Facebook para los estudiantes, entreviendo los tipos de contenidos y la factibilidad de los mismos y el cumpliendo con el objetivo de creación. Se inicia con la pregunta valorativa que comprende el rango de edad del grupo encuestado, el tiempo en cuanto uso de la red social Facebook, y constriñendo la temática en cuanto a la influencia de este espacio universitario para con el alumnado.

\section{Análisis de tabulación}

\footnotetext{
${ }^{10}$ Datos del Anexo 2
} 


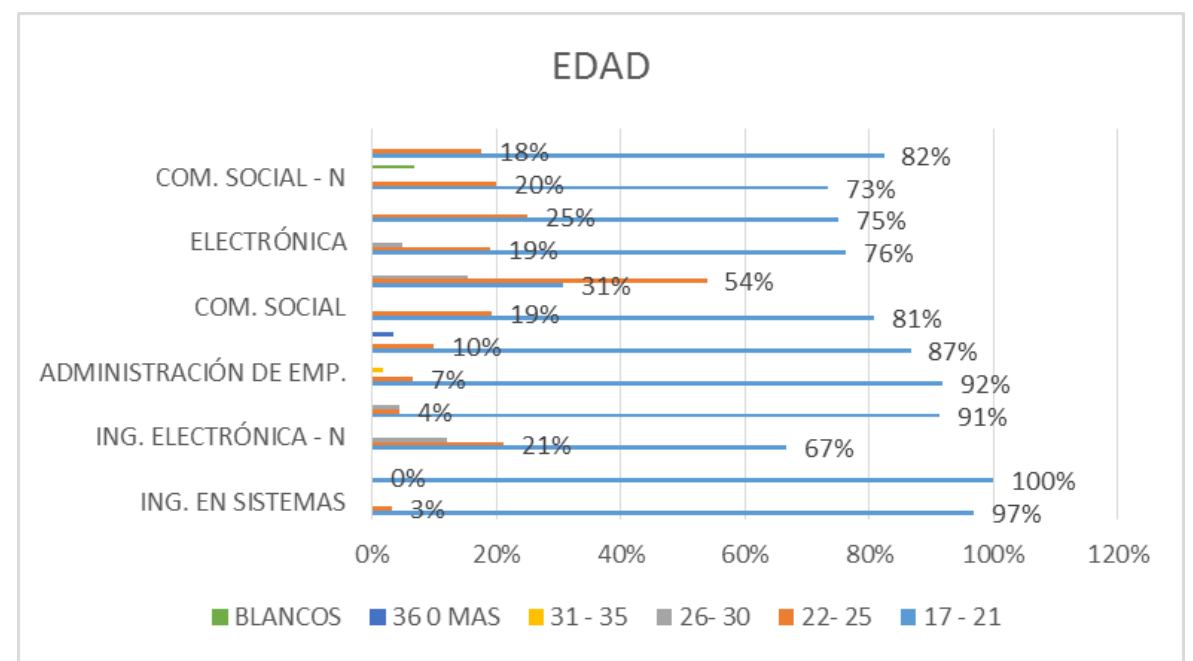

Gráfico 1 Rango de edad de estudiantes de la UPS, Guayaquil Fuente: Elaboración propia

La primera tabla muestra el rango de edad del universo encuestado, entre 17 y 21 años de edad, se comprende que esta edad centra una realidad de continua actualización, frente a la tecnología, las nuevas redes sociales, y las herramientas de comunicación que están en auge.

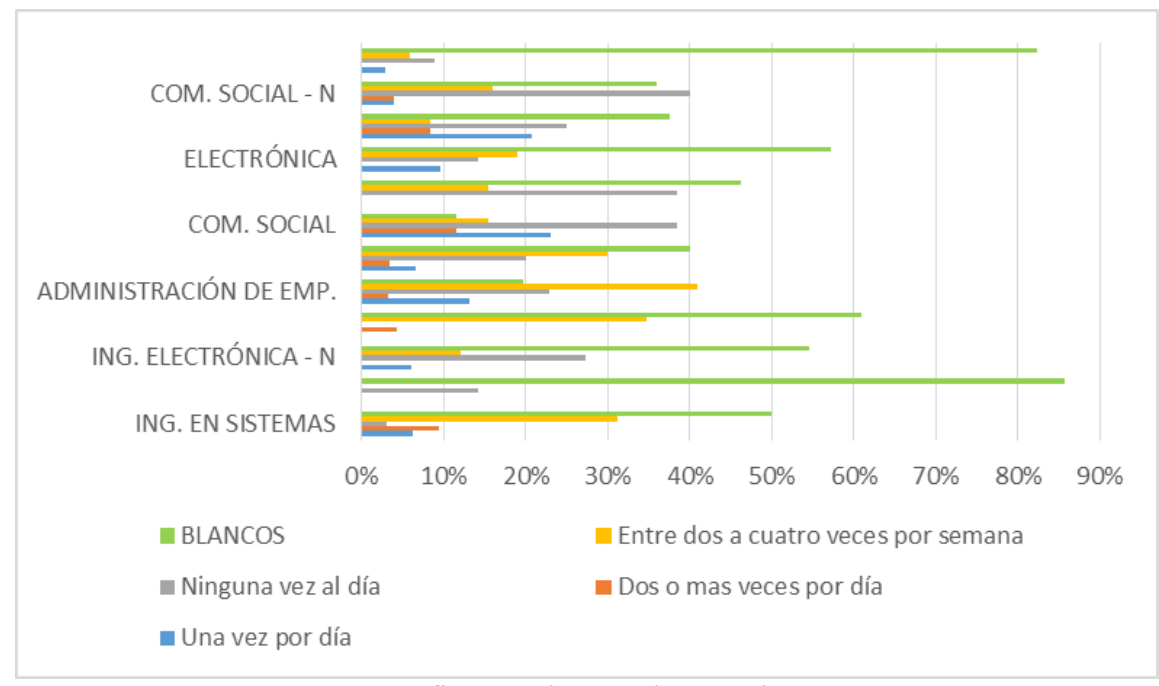

Gráfica 2 Alcance de usuarios

Fuente: Elaboración propia

Se evidencia que los usuarios frecuentan entre 2 a 4 veces por semana la página de la UPSG, creando una interacción entre usuarios e institución considerable para la muestra que se analizó. 


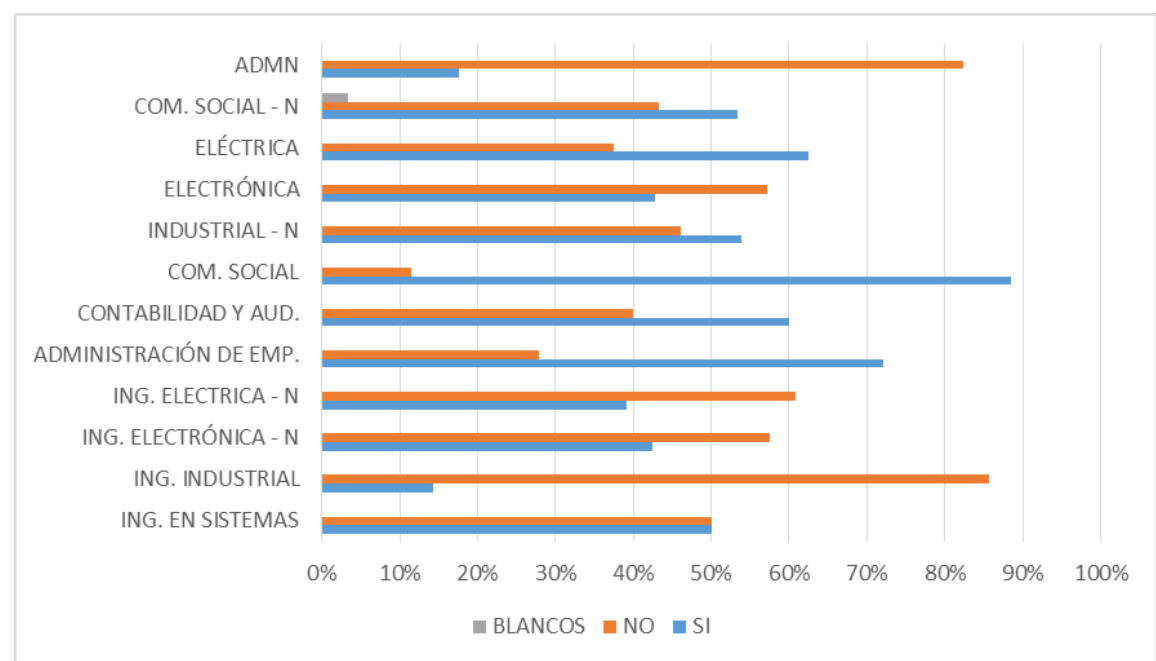

Gráfica 3 Alcance y conocimiento de la página de Facebook institucional Fuente: Elaboración propia

Se determina que la falta de conocimiento de la página presenta un porcentaje elevado en Ingeniera Industrial y Administración de Empresas con más del 80 \%, mientras que la carrera con mayor conocimiento de la plataforma social es de Comunicación Social con un 90 \%.

En Facebook se pudo evidenciar algunos espacios, los cuales no son de administración oficial del personal de la Dirección Técnica de Comunicación y Cultura (Ups Guayaquil), estas fueron creadas por otras instancias dentro de la UPS-G no solo la particularidad de la representación de una carrera - especialidad, sino también, son dirigidas por administradores que no siempre publican realidades fidedignas, sobre acontecimientos académicos veraces.

La puesta en escena de estas dos preguntas, ya tabuladas, nos indica un porcentaje significativo de encuestado del 49\% que no ingresan a la red social Facebook y optativo a ello, su respuesta está en blanco. El caso de Administración de Empresas e Ingeniería Industrial, mostrando como resultado nulo, más de un $80 \%$. Muestra, la misma tabla, con un $35 \%$ en Ingeniería Eléctrica, el ingreso a la red social Facebook se sobre pasa de dos o más veces al día. Por otro lado, en cuanto al conocimiento de la existencia de un espacio en Facebook de la Universidad Politécnica Salesiana- Guayaquil, la Carrera de Comunicación Social responde de manera positiva, con más de un $90 \%$ sobre el conocimiento y existencia de este espacio. Mientras que seis carreras responden con más de un 50\%, al desconocimiento del mismo. 


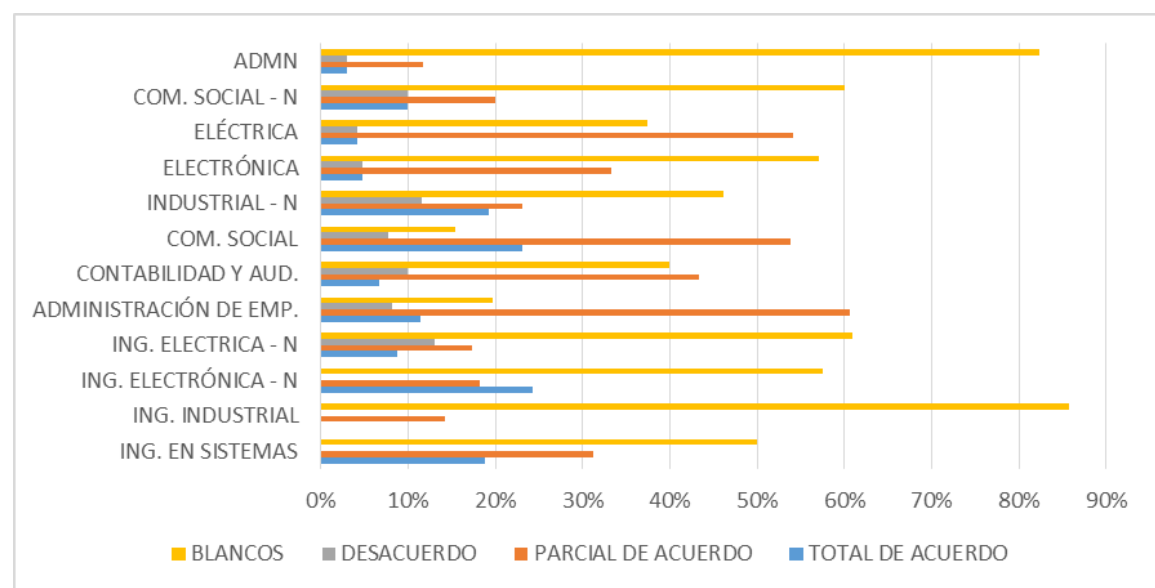

Gráfica 4 Efectividad de la información del entorno universitario Fuente: Elaboración Propia

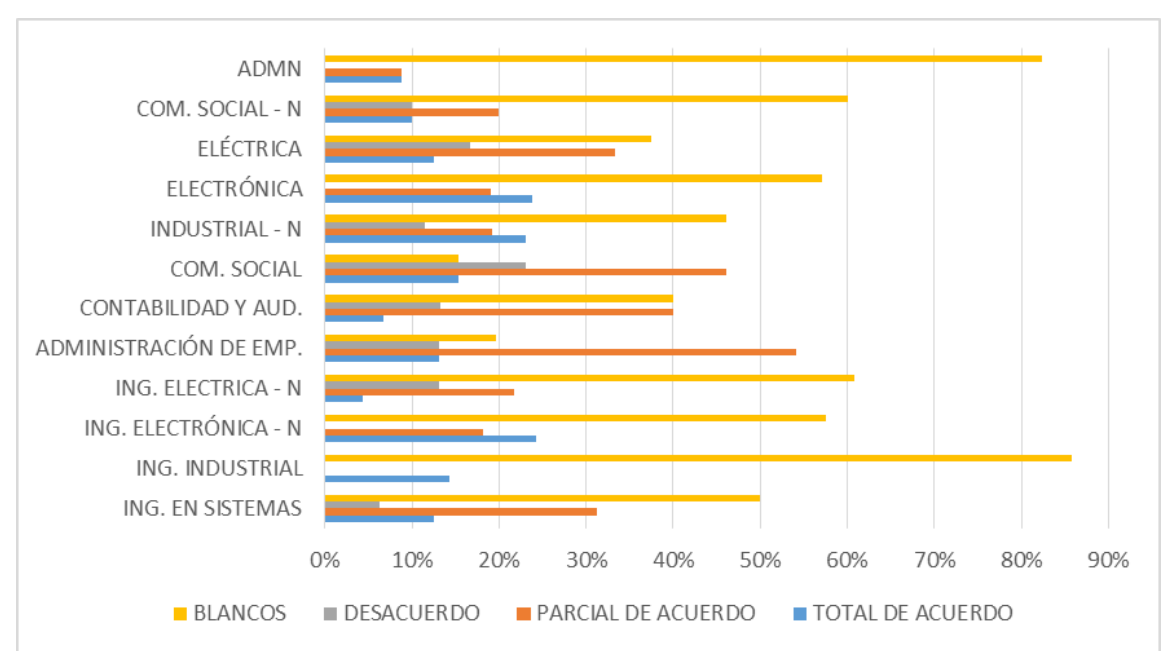

Gráfica 5 Impacto de la información de las actividades universitarias Fuente: Elaboración Propia

En cuanto a las estadísticas, tanto en la gráfica 4 como en la gráfica 5, relacionan la comunicación respecto a la realidad informativa del espacio Facebook de la UPS-Guayaquil, comprendiendo que hay una cifra representativa en cuanto respuestas en blanco, caso que se comprende como negativo para la correcta difusión de la información. Se puede analizar que las respuestas de las dos representaciones para la comprensión están totalmente de acuerdo y llega a un $20 \%$ como alcance de la información hacia los discentes. 


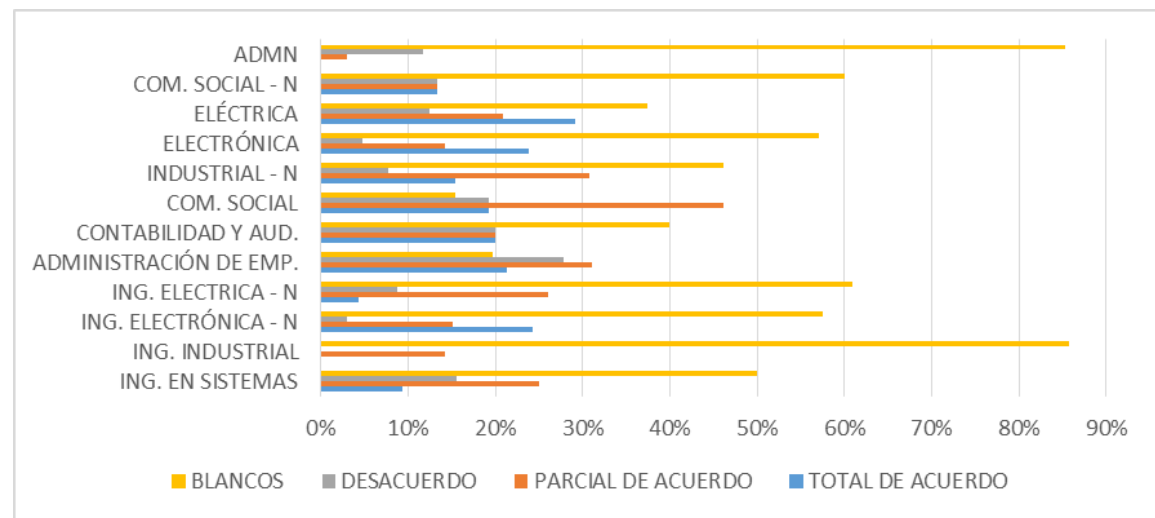

Gráfica 6 Medios y otros recursos informativos

Fuente: Elaboración propia

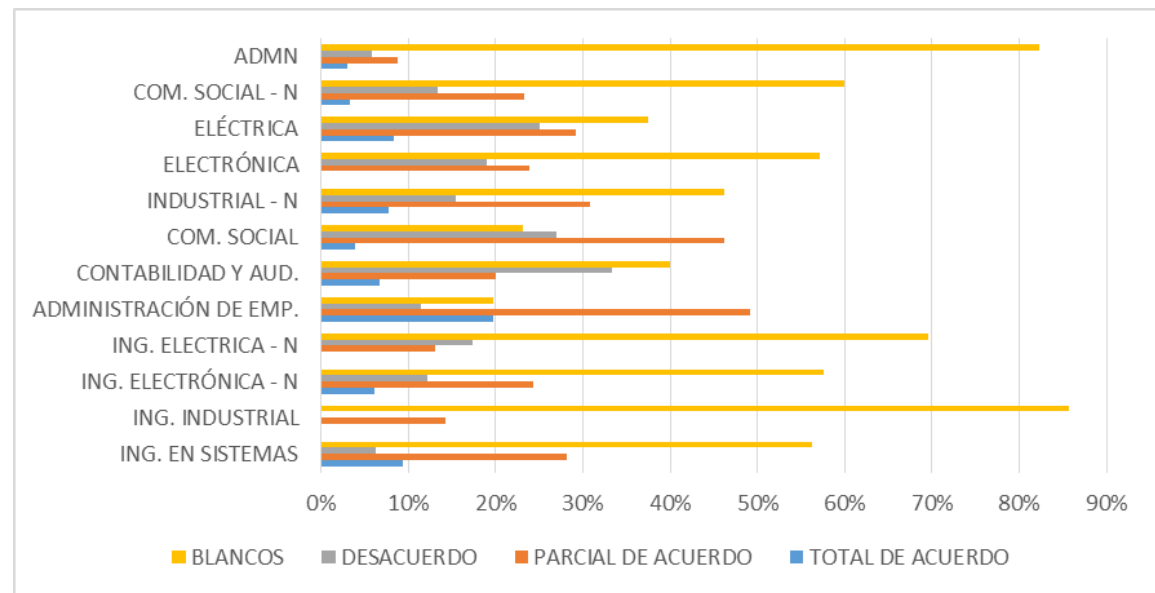

Gráfica 7 Credibilidad de publicaciones

Fuente: Elaboración propia

La respuesta en relación con la gráfica 6 , hace referencia al desconocimiento e interés que se desarrolla para con la información de las actividades, ya que se comprende que incluso, de acuerdo a la tabulación, se comprueba que existe mucha despreocupación por informarse de los acontecimientos institucionales, sabiendo que estos se difunden en varios espacios y de diferente forma. Así mismo se considera parcialmente de acuerdo con un 45\% de respuesta, sobre la verosimilitud de la información que se publica en el espacio Facebook. La posible comprensión de que existen varios espacios en Facebook con el nombre de Universidad Politécnica Salesiana - Guayaquil, puede resultar confuso para la difusión de información fidedigna y para la comprensión de contenidos reales ${ }^{11}$.

\footnotetext{
${ }^{11}$ Mirar Anexo2 - 2.- Otros espacios en Facebook similares a los de la UPS-G.
} 


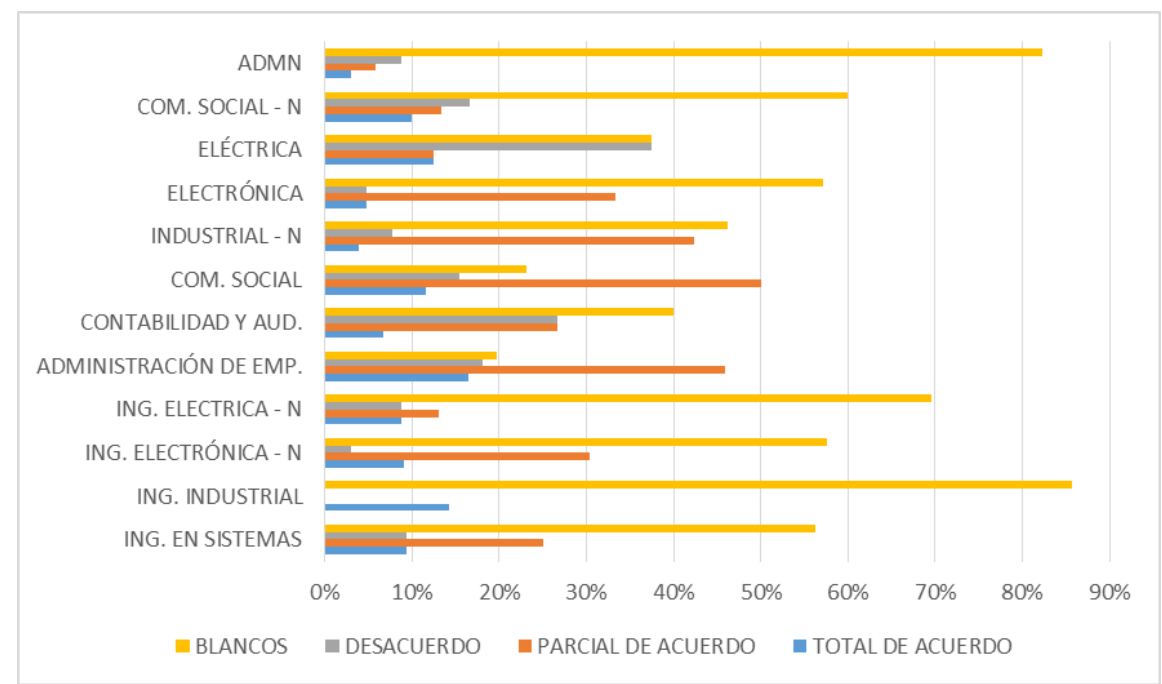

Gráfica 8 Efectividad en información para estudiantes Fuente: Elaboración propia

Aludiendo a la gráfica 8 la comprensión del material informativo que se postea, existe una respuesta de parcial de acuerdo de la información que se publica y actualiza. Observando mediante el análisis que quizás la información que se publica escapa del interés o no es relevante para los estudiantes, considerando el 50\% para la carrera de Comunicación Social y en el mínimo porcentaje para Administración de Empresas con menos del 10\%.

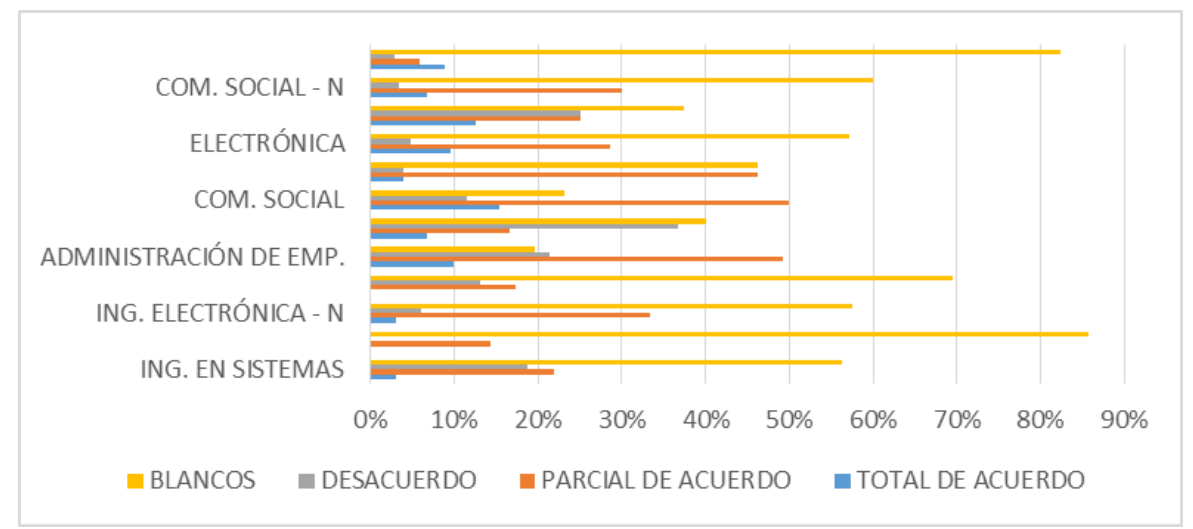

Gráfica 9 Efectividad, información de interés institucional

Fuente: Elaboración propia 


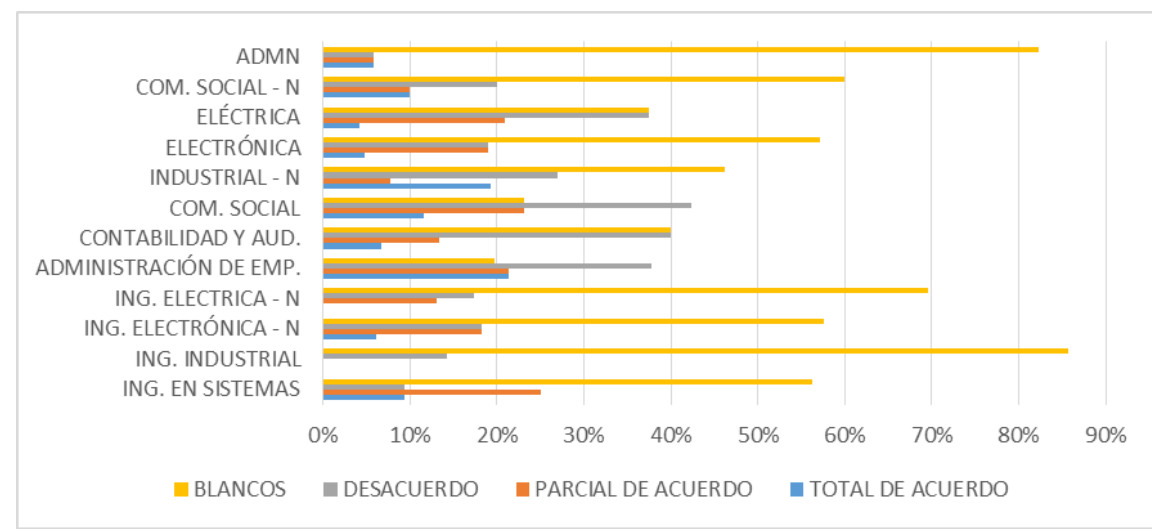

Gráfica 10 Alcance de la plataforma Facebook de la UPS-G

Fuente: Elaboración propia

Referente a la gráfica 10 se analiza la importancia de Facebook como medio de comunicación, que presenta, al ser el modelo gestor de la sociedad en red y de la comunicación, múltiples opciones de interacción y grupos, para la multitud de personas que ingresan a diario.

Por ende resulta interesante comprender como es que siendo el espacio de Facebook de la UPS-G ${ }^{12}$ una página tan popular, desarrolle respuestas muy bajas para con el interés en la misma.

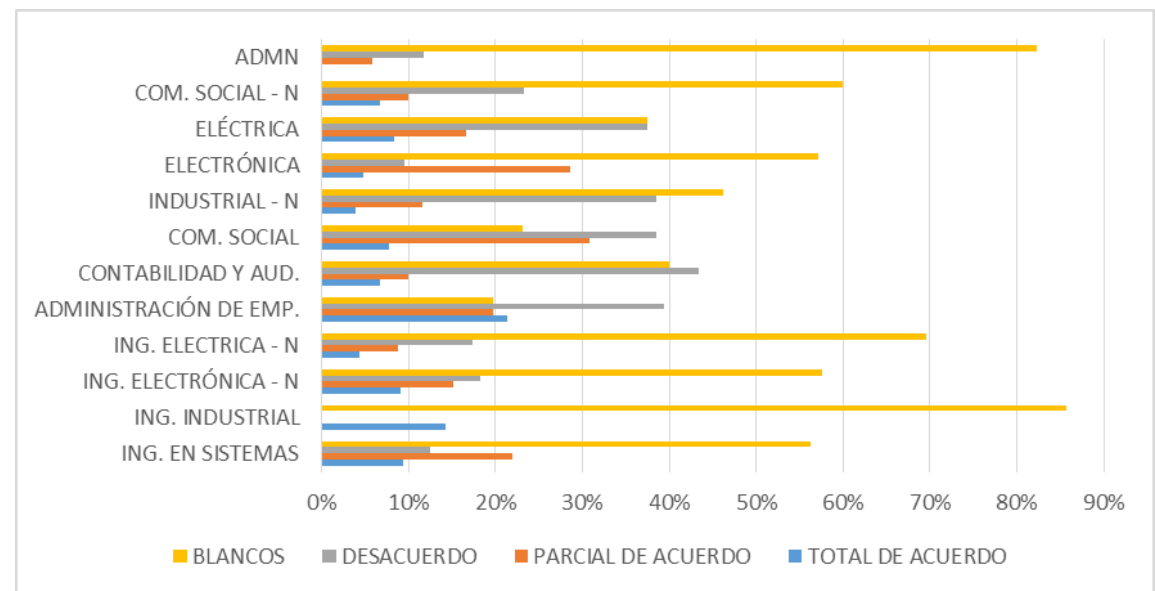

Gráfica 11 Impacto de interacción de la plataforma Facebook Fuente: Elaboración propia

Así mismo se tiene dudas en la gráfica 11 en desarrollar espacios de interacción y publicaciones libres, que generan diálogos para promover de mejor forma la comunicación, ya que hay más de un $40 \%$ en desacuerdo para con la aportación de generación de dialogo e interacción entre los 8031 miembros de este espacio.

Se puede intuir el desinterés por este punto debido a todo lo anteriormente planteado, dudas por la información fidedigna, poco interés por la información de la institución y más.

\footnotetext{
${ }^{12}$ Anexo2 - Con más de 8031 me gusta, manera que mide Facebook a los visitantes frecuentes de esta plataforma.
} 


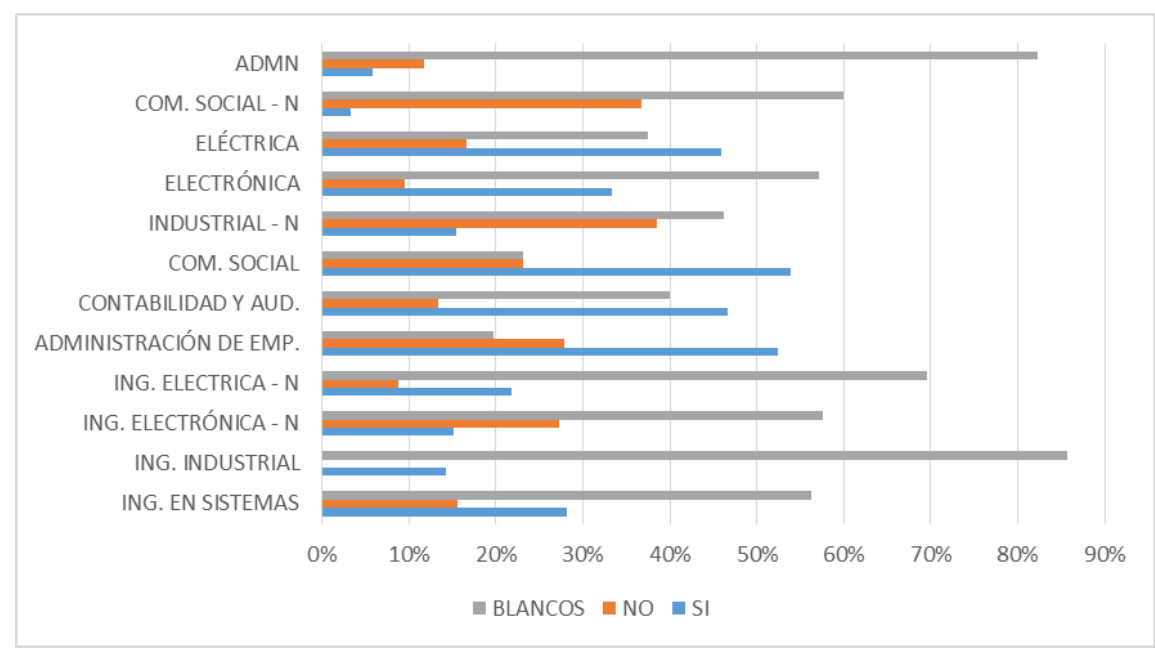

Gráfica 12 Credibilidad y contribución de la información Fuente: Elaboración propia

Pese a todo lo anteriormente planteado, y el elevado porcentaje de personas que dejaron en blanco sus encuestas, esto significa un aspecto negativo o poco importante para los sujetos encuestados, que como criterio general hay un porcentaje alto de aprobación frente a la buena administración en el manejo del espacio Facebook de la UPS-Guayaquil, mostrando más de un $50 \%$ de aprobación positiva para con el buen manejo de este medio.

A la final los datos, postulan que pueden enfrentarse a múltiples campos de estudios, sobre interacciones sociales, modos de información, entre otros. El universo encuestado, evidencia el avance y los cambios que se postulan en los procesos de comunicación e interacción, comprendiendo la relevancia que existe en la actualidad sobre el hecho de poder generar lazos sociales mediante internet y la web 2.0. Se entiende que el universo de la investigación tiene rasgos relevantes por las redes sociales, pero muy bajo interés en el ejemplo desarrollado, donde puede comunicar la institución (UPS-G) directamente al estudiante.

\section{Discusión}

La influencia de las redes sociales como medio de comunicación, más a la par de este proceso, puede también crear una generalidad errónea. Para ello es fundamental comprender el procesamiento de la información y el desarrollo de la investigación en sí, incluso para generar, como se planteó con anterioridad, una correcta herramienta para futuros trabajos con esta tónica. El problema científico plantea un cuestionamiento ineludible sobre el impacto de la red social Facebook para contribuir a la credibilidad de la información a los estudiantes de la Universidad Politécnica Salesiana sede Guayaquil, sin embargo la efectividad de esta herramienta no se sitúa en el plano operacional y de registro de vistas sino más en el plano de compartir y generación de contenidos con significación, por lo cual es trascendente dilucidar cuán efectiva es la comunicación que se efectúa a través de la plataforma, y cuál es el alcance que tiene hacia los usuarios, revisando los contenidos que se postean, analizando si la información que se manipula es de manera adecuada y confiable para los estudiantes que pueden convertirse en prosdumidores. Para ello, la incógnita a responder va de la mano con la realidad social de la plataforma Facebook de la UPS-G, que pretende dar con la respuesta más cercana a lo que aspira la presente investigación. 
Las redes de la información en la realidad nacional (realidad universitaria): Al determinar el consumo y alcance de la comunicación e información en la realidad ecuatoriana, refleja cómo estamos inmiscuidos en la terminología de Wallerstein ${ }^{13}$ definiéndola cómo las periferias dentro de un sistema mundo moderno capitalista, o en términos tecnológicos informativos, una red global, para la cual, la participación y la realidad del país no genera una mayor incidencia en cuanto al cambio del paradigma comunicativo, entrando en la conformidad obligatoria de ser participante de múltiples opciones que ofrece el internet o más claramente la web 2.0.

El menosprecio no es temática palpable, sino una realidad evidente que muestra el hecho de la existencia un nuevo sistema de comunicación que cada vez habla más un lenguaje digital universal, está integrando globalmente la producción y distribución de palabras, sonidos e imágenes de nuestra cultura y acomodándolas a los gustos de las identidades y temperamentos de los individuos (Castells, 1996)

Tomando en cuenta la oportunidad de destacar los factores educativos, no solo como servicio sino como identidad, la Universidad Politécnica Salesiana, sede Guayaquil, debería cimentar las fortalezas de esta red social como un espacio que legimite contenidos oficiales, pero además propicie mayor identificación e interacción con los estudiantes. Sin embargo cabe precisar que este proyecto universitario en la plataforma Facebook, se encamina a la realidad de su evolución comunicativa y desploma de manera acelerada el proceso tradicional de información.

Básicamente, la incorporación de la universidad a la plataforma Facebook, busca, no solo crear un proceso de identidad, de reconocimiento y pertenencia en la institución, sino, y de manera más exacta y como objetivo específico, de interacción con la comunidad universitaria, en el cual la comunidad universitaria considera oportuna su participación, como plataforma libre, a propuestas de actividades y cambios; del mismo modo un propósito sustancial desarrolla la idea de mantener a los estudiantes informados sobre las actividades que se van suscitando de manera local. No obstante, los informativos estudiantiles universitarios existieron, al parecer, desde la creación misma de la institución, de la mano de esa necesidad de comunicarse expuesta con anterioridad, solo que su recurso a lo sumo de su información continua, solo destacaba en repositorios mensuales de información trascendental, prueba de la que existe hasta la actualidad, con medios como el Noti-UPS. Contextualizando también este planteamiento, se logra comprender que, en la creación de la Universidad Politécnica Salesiana en el año de 1995 la información no tenía la capacidad, ni Ecuador ni en ningún país de América latina y el Caribe, de ser inmediata, y que a la par de los avances tecnológicos se fueron gestando proyectos. Para Flores (2009):

La información necesaria para el conocimiento público, no pretende restringir, el compendio de las actividades más trascendentales que puedan suceder y representar en medios informativos, de interacción sobre la misma y una presentación de todo lo que se puede vivir en la cotidianidad. (...) en los 80's desarrolló el proceso de

\footnotetext{
${ }^{13}$ Wallerstein: principal teórico del análisis de sistema - mundo.
} 
«mediamorfosis ${ }^{14} »$, acuñado por Roger Filder que define el cambio de los medios de comunicación, actualmente atravesamos un proceso de «infomorfosis $\mathrm{s}^{15}$ », que se define como: cambio de los mensajes informativos independientes (p.78).

Con las premisas expuestas, comprender que las redes sociales permiten este desplazamiento de particulares, propiciando múltiples elecciones que inciden en una "pseudoidentidad" ${ }^{16}$ de las cosas que adquiere su interés y que la construcción del imaginario en las redes sociales vs la realidad del sujeto: El evidente progreso comunicacional en las alternativas de interés individual no se hacen esperar; es así como se van desarrollando sitios para cada gusto y variedad de las personas o grupos particulares. Plataformas en internet fraguan una colectividad y, en busca de la particular información desarrollan entornos de interacción.

La verosimilitud del sujeto del ciberespacio se pone en tela de juicio, por el hecho de que la construcción de su realidad es adaptable a los estándares mentales que puede producir la persona, llegando al punto de fabricar un ser ideal, exagerando e incluso sosegando los defectos naturales, dificultando con esto la verdad y la identidad del sujeto; siendo las redes sociales una oportunidad de crear versiones fantásticas de sí mismos que en muchos casos, rebosan virtudes y carecen de defectos (Aguilar Rodríguez \& Said Hung, 2010), destacando en la quimera de su autenticidad los niveles de estudio, el tipo de institución a la que pertenece, el tipo de trabajo, los gustos e intereses particulares, artísticos, musicales, y más.

Entonces, es innegable que la realidad de mostrar un imaginario de sí, va de la mano de todos los intereses que se pueden colocar de la persona que interactúa por estos nuevos medios sociales. Cuando se menciona, el hecho de presunciones de estudio, es importante poder relacionar esta idea con el desarrollo, no solo con el imaginario del sujeto, sino de su distinción como un profesional.

Ninguna institución escatima la oportunidad de interactuar con espacios identitarios para los usuarios de Facebook, tanto en la realidad de su distinción, como en la recopilación de recursos (recuerdos, invitaciones, noticias, y más) de información inmediata. Con relación, no a la distinción del sujeto para con una institución, sino a lo que las instituciones pueden comunicar o no al sujeto, se levanta este estudio, el nivel de influencia que pueden desarrollar las publicaciones e interacciones se generan con los usuarios que se identifican con la empresainstitución de su interés.

Para entender de mejor forma este punto, se entenderá por patrón que prefigure la ejemplificación del modelo comunicativo de una institución. Y con ello investigar la influencia que se produce en el grupo particular, este caso estudiantes de la UPS-G, considerándolos al aplicar la entrevista como el grupo de interés.

\footnotetext{
${ }^{14}$ Mediamorfosis: Es la transformación de los medios de comunicación, que generalmente es resultado de la interacción compleja entre las necesidades percibidas, las presiones políticas y de la competencia, y de las innovaciones sociales y tecnológicas.

${ }^{15}$ Infomorfosis: Es la transformación de la información en los medios de comunicación, plataformas.

${ }^{16}$ Pseudoidentidad: La pseudo-identidad es una variación del status normal de la identidad, y se produce debido al razonamiento fundamentado en la comparación, lo cual conduce a la esclavitud de la persona debido a que no puede manifestarse con libertad como ella misma es, pues ha generado en sí misma otros parámetros diferentes a lo que es su realidad.
} 
Es necesario comprender que un estudio no data de la realidad de la identificación del individuo por una institución, sino algo más allá del nivel de influencia que se presenta en él, y que al avanzar la investigación ésta podría eliminar una quimera de simple distinción y reconocimiento por influencia.

\section{Conclusión}

La evolución de las formas comunicativas y de información, resulta ser un proceso inconmensurable que puede contarse de manera histórica, pero al mismo tiempo es impredecible para con su futuro. En la actualidad la web 2.0 es una alternativa de socialización que genera un "mundo de las ideas" fuera del campo material e incluso real. La sociedad se gesta en internet, las redes sociales, al igual que en su tiempo la prensa, la radio y la televisión, han revolucionado, no solo la forma de comunicarse sino la forma de interactuar, de distribuir información, de opinar. Así en la trayectoria de los años, se mira como el internet cambia, incluso, la forma de mirar al mundo, mejorando innegablemente la comunicación, llevándola al proceso de inmediatez, escatimando distancias, esto se ejemplifica de mejor forma con la producción de las redes sociales, estas tienen como teleología, saber que las personas no solo se comunican, sino que también interactúan.

Modificando estos puntos tanto a nivel individual como a grandes escalas, en el caso de las empresas, instituciones y más; el análisis que se puede presentar sobre las redes sociales, más allá de juicios axiológicos, moralistas o de los pros y contra que presenta esta herramienta, modifica los esquemas de relaciones entre individuos ${ }^{17}$. El cambio comunicativo - social presenta un nuevo giro, incluso, a los procesos del mercado y publicidad de las instituciones y empresas. Las redes sociales tienen la identificación de ser de gran ayuda para los individuos y las instituciones - empresas, llegando incluso a comprender que el sujeto se deja influir por las mismas.

Más, se puede deducir que, de este proceso, hay dos caras de la moneda. En un lado se encuentra el hecho de lo que el sujeto construye para mostrar abiertamente su identidad, gustos, intereses, estudios, trabajo y más. Y el otro lado de la moneda muestra el hecho de que por más que se geste la sociedad en la red, no hay forma de comprender cuan influenciable pueden llegar a ser los interese de los sujetos. Esto hace referencia a la encuesta planteada, sabiendo que hay un bajísimo interés e influencia, de lo que presenta el espacio en Facebook de la UPS-G para los estudiantes, sabiendo que hay mucha información de calidad que se publica en la misma ${ }^{18} \mathrm{e}$ informaciones que se renuevan y se concatenan incluso con sitios oficiales ${ }^{19}$.

\footnotetext{
${ }^{17}$ Se comprende que las redes sociales influyen de manera positiva, cuando se usan con recato y moderación, ya que es un medio por el cual te puedes comunicar, no solo para charlar y planear eventos sociales, sino también para hacer tareas y trabajos (Morales 2011)

${ }^{18}$ Se postean en UPS Guayaquil noticias que se publican en el sitio web institucional www.ups.edu.ec, creando un enlace entre ambas cuentas y alcance credibilidad para quienes conviven día a día en la UPSG. ANEXO2

${ }^{19}$ En la actualidad, bajo la administración del MSc. César Andrade Martínez, lleva el mismo esquema de informar a la comunidad universitaria, no sólo la gestión académica y administrativa con imágenes y textos, e incluye productos audiovisuales que genera el UPS Channel TV, Canal de YouTube: https://www.youtube.com/user/UPSCHANNELTV, para llegar de una mejor manera con información real y oportuna que pone en evidencia la gestión dentro de las instalaciones. ANEXO2
} 
Pareciera que el problema de interacción, presenta varias aristas para con este análisis, por un lado esta lo anteriormente proyectado e intuido, por las estadísticas, que intuye una falta de interés y una aceptación del espacio por prestigio de la institución; más, por el otro lado, se comprende que hay una falta de realidad en cuanto a la divulgación, escasa publicidad y, pese a la buena puntuación en cuanto a la administración, de la pregunta novena de la encuesta, una falla por parte de la administración de este espacio.

En base a las encuestas se puede evidenciar que existe cierto desconocimiento sobre UPS-Guayaquil (cuenta de educación), al preguntar a estudiantes si la conocían, cada uno de ellos manifiesta que "existen varias cuentas de cada dirección de carrera" eso quiere decir que en la actualidad, al existir siete carreras hay un espacio por cada una de ellas.

Se puede tener en cuenta, en la parte final del análisis de los datos estadísticos presentados, como los estudiantes al elegir espacios que les interesen, comprendiendo la importancia de verse inmiscuidos y de interactuar en las redes sociales mostrándose este como el espacio en donde se gesta la sociedad en la actualidad, solo utilizan sus elecciones para generar una carta de presentación al mundo en el que se están manejando; sin importar muchas de las veces, que se está eligiendo para mostrarse al mundo, corroborando el hecho de que los estudiantes solo construyen su información de acuerdo a su interés y al imaginario que desea formar de sí mismo.

Finalmente se puede colegir que lo que resulta importante darle una respuesta al proceso planteado en toda esta investigación, entregando como resultado que la influencia, del espacio Facebook de la Universidad Politécnica Salesiana sede Guayaquil, es mínima por la deducción de los factores que se han postulado con anterioridad. Esto puede comprender que el imaginario de la creación de un sujeto, en las redes sociales, puede proyectarse de manera figurativa el ideal del mismo, ya que mucho muy poco le puede importar lo que suceda en torno (a lo que se publique, lo que se comente o lo que se desarrolle) a lo que el sujeto selecciona como contenido de su pseudo - identidad. Así mismo, se extiende una larga reflexión y reto sobre los nuevos procesos de difusión e información de este espacio para los estudiantes universitarios.

\section{Bibliografía}

Aguilar Rodríguez, D. E., \& Said Hung, E. (2010). Identidad y subjetividad en las redes sociales virtuales: caso de Facebook. Zona Próxima, núm. 12, 190-207.

ALEXA. (s.f.). Alexa. Obtenido de Alexa: www.alexa.com/topsites/category/World/../Base_de_datos

Augé, M. (2000). Los no lugares - Espacios del anonimato. Barcelona: Editorial Gedisa, S.A.

Campos, F. (2008). Las redes sociales trastocan los modelos de los medios de comunicación tradicionales. Revista Latina de Comunicación Social 63, http://www.revistalatinacs.org/_2008/23_34_Santiago/Francisco_Campos.html

Castells, M. (11 de 3 de 2001). (http://www.areacomunicacion.com.ar/text/3_006.htm, Entrevistador)

Castells, M. (2001). Internet y la Sociedad Red . 1. 
Castells, Manuel. (1996). La era de la información. Economía, sociedad y cultura. Vol. 1. México: Siglo XXI.

Chalmers, A.-F. (1987). Qué es esa cosa llamada ciencia? Madrid: Siglo Veintiuno Editores.

Corporacion Asesoría Económica \& Marketing. (2009). Corporacion Asesoría Económica \& Marketing. Obtenido de Corporacion Asesoría Económica \& Marketing: http://www.corporacionaem.com/tools/calc_muestras.php

Drucker, P. F. (2001). Detrás de la revolución de la información. Revista La Factoría, octubre-enero, (13), http://seinest.com/pdf/artigo2.pdf

El Comercio. (17 de Agosto de 2014). El uso de Internet en Ecuador creció 11 veces en siete años. El Comercio, págs. http://www.elcomercio.com/tendencias/ecuador-internet-datos-tecnologiausuarios.html

Flores, J. M. (2009). Nuevos modelos de comunicación, perfiles y tendencias en las redes sociales. DOSSER, 74-81.

Focoult y el Poder. (s.f.).

García, M. (2002). Foucault y el poder. México: Universidad Autónoma Metropolitana.

INEC. (2013). instituto nacional de estadísticas y censos. Tecnologías de la Información y Comunicaciones (TIC'S) . Ecuador: http://www.ecuadorencifras.gob.ec/documentos/webinec/Estadisticas_Sociales/TIC/Resultados_principales_140515.Tic.pdf

LA NACION. (Viernes 04 de Octubre de 2013). Cuáles son los sitios web más populares del mundo en cada país. Obtenido de http://www.lanacion.com.ar/1625907-cuales-son-los-sitios-webmas-populares-del-mundo-en-cada-pais

March, A. (2012). La digitalización de la comunicación humana: alteraciones y cambios en la percepción. Creación y Producción en Diseño y Comunicación, http://fido.palermo.edu/servicios_dyc/publicacionesdc/vista/detalle_articulo.php?id_articulo= 8721\&id_libro=416

Martínez, M. (2009). La investigación sobre comunicación en España.: Evolución histórica y retos actuales. Revista Latina de Comunicación Social., (64), 1.

OII. (09 de 10 de 2015). Informatios Geographies. Obtenido de http://geography.oii.ox.ac.uk/?page=home

Pérez, R., \& Porras, Y. (2005). La Complejidad En El Marco De Una Propuesta Pluriparadigmática. Tecné, Episteme y Didaxis No. 17, 104-116.

Wallerstein, I. M. (2005). Análisis de sistemas-mundo: una introducción. México: Siglo XXI.

Wave 7. (2013). Cracking The Social Code - The Story of wy. Chile: Curiosity Works. 